\title{
Current status of research and application in vascular stents
}

\author{
QI PengKai ${ }^{1,3}$, YANG Ying $^{1,3}$, MAITZ F Manfred ${ }^{2,3} \&$ HUANG Nan $^{1,3^{*}}$ \\ ${ }^{1}$ Key Laboratory of Advanced Technology of Materials of Education Ministry, Southwest Jiaotong University, Chengdu 610031, China; \\ ${ }^{2}$ Max Bergmann Center of Biomaterials Dresden, Leibniz Institute of Polymer Research Dresden, Dresden 01097, Germany; \\ ${ }^{3}$ School of Materials Science and Engineering, Southwest Jiaotong University, Chengdu 610031, China
}

Received April 8, 2013; accepted June 18, 2013; published online August 12, 2013

\begin{abstract}
Cardiovascular diseases have been the leading cause of death in modern society. Using vascular stents to treat these coronary and peripheral artery diseases has been one of the most effective and rapidly adopted medical interventions. During the twenty-five years' development of vascular stents, revolutionary cardiovascular stents like drug eluting stents and endothelial progenitor cells capture stents have emerged. In this review, the evolution of vascular stents is summarized, aiming to provide a glimpse into the future of vascular stents. Advanced designs, focusing on the investigations of new substrates, new platforms, new drugs and new biomolecules are currently under evaluation with promising clinical studies. The concept of "time sequence functional stent" has been raised in this paper. It presents anti-proliferative properties in the first phase after implantation and subsequently support endothelialization. It also shows long-term inertness without release of toxic ions or toxic degradation products. The success of this concept is briefly presented with a clinical study in this model stents.
\end{abstract}

bare metal stent, drug eluting stent, biodegradable stent, EPC-capture stent, endothelialization

Citation: Qi P K, Yang Y, Maitz F M, et al. Current status of research and application in vascular stents. Chin Sci Bull, 2013, 58: 4362-4370, doi: 10.1007/ s11434-013-6070-1

Cardiovascular diseases (CVDs), leading major reasons of death worldwide, are a class of diseases caused by disorders of heart and the blood vessels. These disorders, which are related to a process called atherosclerosis, include numerous problems, for example the accumulation of lipid deposition and lipid-laden macrophages in atherosclerotic plaques. The primary trigger for arterial thrombosis is the rupture of such a plaque. Thus, the altered blood vessel wall interferes with the blood to flow through and increases the risks of heart attack [1]. According to statistics published by the world health organization in 2012, the annual number of deaths due to CVDs will increase from 17 millions in 2008 to 25 millions in 2030 [2]. Applying vascular stents to treat the associated coronary and peripheral artery diseases has been one of the most effective and rapidly adopted medical interventions.

Vascular stents are small expandable tubes which are

*Corresponding author (email: nhuang@263.net) mounted onto a balloon catheter, inserted and expanded at the narrowed section of the vessel, acting like a stabilizing scaffold for the artery to maintain the patency of the vessel in order to treat narrowed or weakened arteries in the body [3]. At present, the implantation of stents has been utilized as a minimally invasive treatment for CVDs and becomes the most common percutaneous coronary intervention. During the decades' development, several generations of stents have been designed with a growing knowledge of the interaction among stent, blood and endothelium. The first generation stent was named bare metal stent (BMS). Although numerous reports have suggested the increasing incidences of in-stent restenosis (ISR) after the implantation of BMS, it is still widely used in clinic today [4]. Drug eluting stent (DES), a revolutionary device to address the problem of ISR, was developed by coating a drugs-loaded polymer onto the BMS. Despite the success of DES to eliminate the ISR, long-term safety and efficacy are questioned due to the late stent thrombosis (LST) reported in 
numerous of clinical trials [5]. It has been demonstrated that the polymer coating caused persistent arterial wall inflammation and the drug delayed the vascular healing. Afterwards, scientists and biomedical engineers made every effort to construct novel stent systems to solve problems occurring in clinic. The ongoing developments of DES concern the core and the coating of the stent as well as the eluted drugs [6]. Meanwhile, novel concepts of stents are raised up such as the biodegradable stents (BDS) and endothelial progenitor cell (EPC) capture stents. Some of these products are currently in clinical evaluation and the outcomes of the studies are highly expected. In this paper, the evolution of vascular stents is discussed and new stent techniques are highlighted. Also, the concept of "time sequence functional stent" is described in detail.

\section{Bare metal stents}

The original strategy of percutaneous balloon dilatation dated back to 1971 , at which time the first percutaneous transluminal coronary angioplasty (PTCA) was performed in patients. By passing a deflated balloon and expanding it through a catheter to the vessel, this technique can open the artery and compress the plaque against vessel wall. After 2 years, there was an overall patency rate of 70\%-80\% [7]. In 1987, Sigwart et al. [8] adopted intravascular stents to prevent occlusion and re-stenosis after transluminal angioplasty. Unlike the percutaneous balloon dilatation, this meshed cylindrical medical device, coronary vascular stent, serves as a mechanical support to expand the narrowed vessel wall temporarily or permanently, preventing elastic recoil and vascular negative remodeling which are the common side effects of balloon angioplasty.

Currently, over dozens of bare metal stents are available in the market. The characteristics for an ideal stent substrate are supposed to include: excellent mechanical properties for intervention and expansion, adequate radiopacity for visualization during angiography, good hemocompatibility for implantation without much side reactions [9]. Generally speaking, commonly used metals for manufacturing are 316L stainless steel (316L SS), cobalt-chromium (Co-Cr) alloy, titanium and its alloy (e.g. Nitinol). Besides, platinum-iridium (Pt-Ir) alloy and tantalum (Ta) are alternatives in some products. Among these stent substrates, 316L SS is the most commonly used metal for stents. There are overall eight coronary stents approved by the US Food and Drug Administration (FDA), most of which are made from 316L SS in both BMS and DES. While considering poor hemocompatibility of the surface and the long-term release of toxic ions such as $\mathrm{Cr}, \mathrm{Ni}$ and $\mathrm{Co}, 316 \mathrm{~L}$ SS stents are prone of ISR, limiting their applications in clinic [10].

Restenosis, which is defined as diameter stenosis of $\geqslant 50 \%$ in the stented area of the vessel, is a multifactorial process primarily because of the vascular trauma and the denudation of endothelium after balloon angioplasty and/or stent implantation [11]. The basic biological processes of restenosis are mainly attributed to the thrombogenicity. Thrombosis involves the activation, adhesion and aggregation of platelets which is dependent on the degree of vascular damages [12]. Meanwhile, presence of vascular injury and foreign materials lead to a disorder of coagulation as well as inflammatory and complement systems. The activated neutrophils and macrophages together with the released cytokines and growth factors accelerate the hyperplasia of smooth muscle cells (SMCs), leading to remodeling of the extracellular matrix (ECM) and initiating smooth muscle cell migration $[13,14]$. The end results of these cascade events are the thrombus and ISR, which are the key points for consideration when novel vascular stents are designed and developed. Therefore, based on the BMS, numerous surface modification approaches and novel concept vascular stents aiming at modulating biological responses and improving the stent performance are developed. To inhibit ISR, some radioactive stents $[15,16]$ and drug eluting stent $[17,18]$ were reported as the effective ones. However, undergoing radiation brachytherapy or using radioactive stents have also shown that proliferation at the stent edges may be associated with higher-than-expected restenosis rates at the stent margins. As more evidence supported that radioactive stents delayed but do not prevented in-stent neointimal hyperplasia [19], scientists gradually paid much more attention on the novel designs of drug eluting stents.

\section{Drug eluting stents}

Among numerous methods for improving vascular stents by surface modification, there is no doubt that the drug eluting stent is the most successful one in the history of stent development. It is considered as a wonderful creation of modern medical engineering that aid physicians and surgeons in the treatment of CVDs. As a revolutionary medical device combining drug and stent, DES shows lower rates of ISR and is statistically superior to BMS. Up to date, there exist two generations of DES. Encouragingly, newer DESs are under research and clinical investigation at the same time [20]. The predominantly applied DESs approved for clinical use are summarized in Table 1.

The first generation DESs are Cypher ${ }^{\mathrm{TM}}$ (Cordis, Warren, New Jersey, USA) and Taxus ${ }^{\mathrm{TM}}$ (Boston Scientific, Natick, Massachusetts, USA), which received regulatory approval from both European Union Conformiteé Européenne (CE) and FDA. Cypher consists of 316L SS platform and two permanent polymer coatings of poly(ethelene-co-vinyl acetate) and poly(n-butyl methacrylate), which are the carrier of sirolimus [21]. The Taxus device also applies a 316L SS substrate and a single polymer/drug mixture layer in which poly(styrene- $b$-isobutylene- $b$-styrene) coating combined with $1 \mu \mathrm{g} / \mathrm{mm}^{2}$ paclitaxel are adopted [22]. Both of these 
Table 1 A summary of some commercial drug eluting vascular stents ${ }^{\mathrm{a})}$

\begin{tabular}{|c|c|c|c|c|}
\hline Categories & Stent name & Substrate material & Coating material & Drug \\
\hline \multirow{4}{*}{ Durable polymer } & Cypher $^{\mathrm{TM}}$ & SS & PEVA, PBMA & Sirolimus \\
\hline & Taxus $^{\mathrm{TM}}$ & SS & SIBS & Paclitaxel \\
\hline & Endeavor $^{\mathrm{TM}}$ & Co-Cr alloy & Phospholipids co-polymer & Zotarolimus \\
\hline & Xience $\mathrm{V}^{\mathrm{TM}}$ & Co-Cr alloy & PBMA, PVDF-HFP & Everolimus \\
\hline \multirow{3}{*}{ Biodegradable polymer } & BioMatrix $^{\mathrm{TM}}$ & SS & PLA & Biolimus A9 \\
\hline & $\mathrm{EXCEL}^{\mathrm{TM}}$ & SS & PLLA & Sirolimus \\
\hline & $\mathrm{NEVO}^{\mathrm{TM}}$ & Co-Cr alloy & PLGA & Sirolimus \\
\hline \multirow{3}{*}{ Nonpolymeric } & BioFreedom $^{\mathrm{TM}}$ & SS & Texture & Biolimus A9 \\
\hline & Yukon $^{\mathrm{TM}}$ & SS & Micropores & Sirolimus \\
\hline & Janus $^{\mathrm{TM}}$ & SS & Reservior & Tacrolimus \\
\hline EPC-capture & Combo Stent ${ }^{\mathrm{TM}}$ & Absorbable polymer & Biodegradable polymer Matrix/CD34 & Sirolimus \\
\hline \multirow{2}{*}{ Fully degradable } & $\mathrm{BVS}^{\mathrm{TM}}$ & PLLA & PLLA & Everolimus \\
\hline & DREAMS & Mg alloy & Bioabsorbable polymer & Paclitaxel \\
\hline
\end{tabular}

a) PEVA, poly(ethelene-co-vinyl acetate); PBMA, poly(n-butyl methacrylate); SIBS, poly(styrene- $b$-isobutylene- $b$-styrene); PVDF-HFP, poly(vinylidenefluoro-hexafluoropropylene); PLA: polylactic acid; PLLA, poly(L-lactic acid); PLGA, poly(lactic-co-glycolic acid); DREAMS, drug-eluting absorbable metal scaffold.

two stents are based on an appropriate combination of metallic platform, permanent polymer and an anti-proliferative drug. In some clinical trials and investigations, the early and midterm safety and efficacy of 1st generation DES are supported. In the 8 months follow up REALITY trials, the first generation durable polymer sirolimus eluting stent (SES) and paclitaxel eluting stent (PES) were reported that the in-segment binary restenosis rate and in-stent late loss are respectively $6.6 \%$ (SES) $/ 11.7 \%(\mathrm{PES})$ and $0.09 \pm 0.43$ $\mathrm{mm} / 0.25 \pm 0.49 \mathrm{~mm}$ [23]. And SIRTAX trials showed the in-segment binary restenosis rate and in-stent late loss are respectively $9.6 \%(\mathrm{SES}) / 11.1 \%(\mathrm{PES})$ and $0.12 \pm 0.36$ $\mathrm{mm} / 0.31 \pm 0.44 \mathrm{~mm}$ after 9 months [24]. However several limitations of SES and PES have emerged. In 2003, Virmani group [25] first reported the incomplete endothelial coverage with focal platelet aggregates and persistent fibrin deposition within the necrotic core at 16 months who received Cypher stent implantation. Their following research also reported the delayed arteries healing in SES which increased the risks of late stent thrombosis, for the antiproliferative agents impede the recovery of endothelium when they suppress the migration and proliferation of SMCs [26]. Although the molecular mechanism of these complex phenomena are still not fully understood, multiple factors including direct toxic effect from the entrapped drug and/or an acute or delayed hypersensitivity reaction from the polymer and/or drug may be involved. The released sirolimus and paclitaxel could increase production of reactive oxygen species and Nitric Oxide bioavailability may be decreased, resulting in impairment of endothelium-mediated vascular relaxation response and endothelial dysfunction in the late stage. The durable polymer from which the drug elutes may also contribute to the adhesion and activation of leukocytes, leading to the local chronic inflammation and hypersensitivity [27]. Besides, most concerns are raised up on the safety of 1 st generation DES especially after discontinuation of dual antiplatelet therapy [28].

Endeavor $^{\mathrm{TM}}$ (Medtronic vascular, Santa Rosa, CA, USA) zotarolimus-eluting stent (ZES) and Xience $\mathrm{V}^{\mathrm{TM}}$ (Abbott Vascular, CA, USA) everolimus-eluting stent (EES) are regarded as the second generation drug eluting stents [29]. Endeavor includes three parts. The substrate is a Co-Cr alloy with good properties in mechanics and radiopacity. The drug-polymer coating is a formation of zotarolimus and persistent anti-fouling phospholipids co-polymer, which constructs an anti-thrombogenic and anti-restenosis surface [30]. Xience $\mathrm{V}^{\mathrm{TM}}$ also adopts the Co-Cr platform. Poly $(n-$ butyl methacrylate) and poly(vinylidenefluoro-hexafluoropropylene) are used in the design, separately acting as an intermediate layer and drug carrier layer [31]. In terms of reduced LST events as well as restenosis rates, clinical results of 2nd generation DES indicated that the long-term safety and promising anti-restenosis efficacy which attributed to anti-proliferative agent, the biocompatible polymer layer and the novel stent frame [32]. There is no conclusive evidence that 2 nd generation DES is superior to 1 st generation DES, as the problems like LST and impaired endothelial healing have been reported in both generation DESs. However, Co-Cr platforms applied in Endeavor ${ }^{\mathrm{TM}}$ and Xience $\mathrm{V}^{\mathrm{TM}}$ stents are better deliverable, more flexible with higher radial strength allowing for thinner strut design than stainless steel 316L SS, presenting better endothelial coverage and less vessel wall injury than Cypher $^{\mathrm{TM}}$ and Taxus $^{\mathrm{TM}}$ stents [33].

It is well known that DES is comprised of three major components as shown in Figure 1. The stent platform scaffolds the vessel to ensure the patency. Polymer coatings deliver the drugs and control the dose and release kinetics through their degradation. The drugs inhibit the neointimal growth and ECM reproduction. Therefore, the current 
strategies of optimizing DES focus on preparing new platforms, new coatings and new techniques of elution [34]. Up to date, different designs of new platforms (e.g. Co-Cr alloy) and new drugs (Biolimus A9) have been evaluated, which opens a new chapter for developing new generation DES. Actually, there are only a few drugs are used in DES, mainly including three categories: mTOR inhibitors (e.g. Sirolimus, Everolimus, Zotarolimus, Biolimus A9); calcineurin inhibitors (e.g. Tacrolimus, Pimecrolimus) and microtubule stabilizer (e.g. Paclitaxel). Besides, dexamethasone, estradiol and genistein are also under investigation for the potential applications in new DES [35,36]. Compared with the development of new drugs and platforms, the methods used to combine drug and stent are far more attracting. Among new DES designs, how to load drug onto the stent surface are paid much more attention, because different drug loading methods not only have an influence on the dose and release kinetics but also on the events of stent-blood interface [37]. Besides, the presence of the drug loading polymer coating has been proved to result in the LST events [38]. Therefore, scientists trend to biodegradable polymers as drug carrier or to develop polymer-free drug loading system.

Numerous DESs with novel drug loading system are undergoing investigation in laboratories and clinical trials, some of which received CE Mark. These are referred as new generation DESs include BioMatix, Nobori, Janus, Yukon etc. [39]. BioMatrix ${ }^{\mathrm{TM}}$ stent (Biosensors, Morges, Switzerland) are based on the SS platform with the thickness of $112 \mu \mathrm{m}$ and biodegradable PLA coating with Biolimus A9. The STEALTH and LEADERS trials showed a remarkable reduction of angiographic restenosis and excellent 9-month clinical safety in all patients [40]. Later, Biosensors developed the nonpolymeric BioFreedom ${ }^{\mathrm{TM}}$ DES based on the BioMatrix ${ }^{\mathrm{TM}}$ stent. It loaded the same mTOR inhibitor, Biolimus A9. BioFreedom ${ }^{\mathrm{TM}}$ is manufactured into micro-structures on the abluminal surface. This polymer-free local drug delivery system not only avoids the adverse effects of degradation products and non-reacted monomer

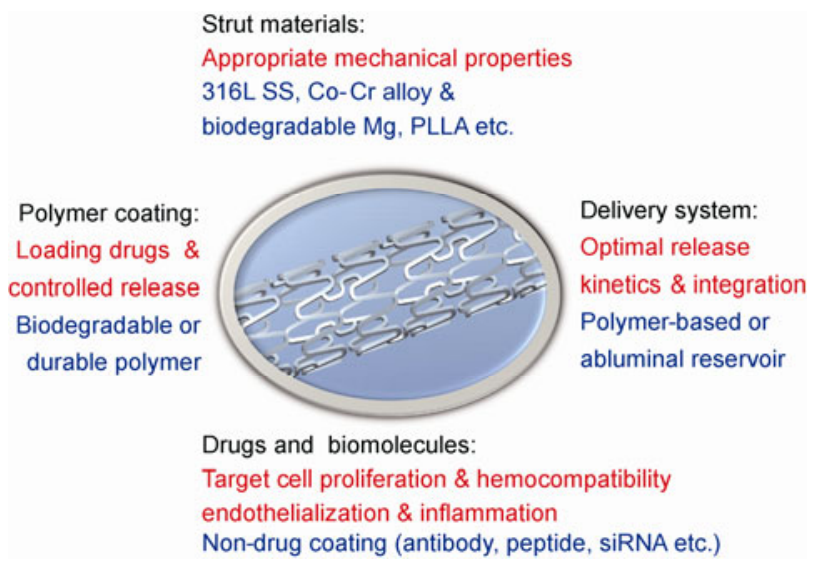

Figure 1 (Color online) Components of drug eluting stents. compounds but also improves healing and integrity of stents [41]. Another commercial stent, Yukon ${ }^{\mathrm{TM}}$ (Translumina, Hechingen, Germany) applies the similar drug loading strategy. Rapamycin is sprayed onto the microporous surface onto a bare SS stent. The micropores on the surface act as a reservoir, allowing the drugs to be released slowly. After the complete drug elution, the remaining microporous surface contributes to the adhesion and migration of endothelial cells [42]. Registry data suggested that the implantation of Yukon ${ }^{\mathrm{TM}}$-DES was feasible and safe, but binary restenosis and target vessel re-vascularization were frequently observed perhaps due to the roughness of surface [43].

\section{Biodegradable stents}

Although DES have obtained great success in interventional surgery, late thrombosis, delayed endothelialization, mechanical mismatch as well as the local hypersensitivity and inflammation were still considered as potential risks [44]. The fundamental cause of these phenomena lies on the fact that stent is indeed a foreign object within the vessel. A promising approach to overcome these limitations is the application of BDS, which can be also considered as bioabsorbable stents [45]. Therefore, BDS is envisaged to support the vessel wall temporarily for about 3-6 months after implantation, during which the stabilizing function of the stent is required. Then the vessel wall will be sufficiently reorganized and BDS could be degraded or absorbed following the healing process. Biodegradable cardiovascular stents can be made of both polymers (lactic acid, glycolic and caprolactone families) and metals (Mg-based or Fe-based alloys) [46]. The most frequently used cardiovascular polymer in the current biodegradable stents is PLLA, which is metabolized into lactic acid, carbon dioxide and water ultimately [47]. It is a recent idea that the metals can be identified as biodegradable materials. In terms of mechanical properties and low toxicity, pure magnesium, iron and their alloys can be applied in designs of vascular stents [48].

The Igaki-Tamai ${ }^{\mathrm{TM}}$ stent (Igaki Medical Planning Company, Kyoto, Japan) is the first BDS which was developed by Tamai et al. [49] in 1999. The total stent is comprised of PLLA and a zig-zag helical coil design with straight bridges. It is worth mentioning that each end of the stent has been implanted gold markers to confer the polymer stent radiopaque. Long-term safety of the Igaki-Tamai stent was confirmed recently according to the clinical outcomes of acceptable rates of major adverse cardiac events and scaffold thrombosis, without stent recoil and vessel remodeling [50,51]. Another two representative fully BDS are named BVS $^{\mathrm{TM}}$ and REVA ${ }^{\mathrm{TM}}$. The BVS ${ }^{\mathrm{TM}}$ (Abbott Vascular, Santa Clara, Calif, USA) everolimus eluting stent adopts PLLA as the strut coated by $\operatorname{poly}(D, L$-lactide $)$ loading everolimus. It possesses the properties of inhibiting neointimal hyperplasia 
like DES and the requirement of disappearance once the stent has fulfilled its task. Whereas PLLA may need 24 months or more to degrade, mismatching the ideal implanting process of integrity for the first 6-12 months and totally degraded after 12-24 months. This kind of polymeric stent may lack sufficient radial force initially, and further decreases with bioabsorption, resulting in recoil of the stent and malapposition at follow-up [52]. The accumulated acidity production caused by the degradation of PLLA also lead to the local chronic inflammation and hypersensitivity like the adverse effect of durable drug loading coating in 1st DES. A prospective, single-group, open-label study was carried out among 30 patients. At 2 years, the in-stent late loss of $0.48 \mathrm{~mm}$ and a diameter stenosis of $27 \%$ are shown in angiography, which do not differ from the findings in 6 months. Consequently, the BVS stent showed excellent clinical safety with no cardiac deaths, ischaemia-driven target lesion re-vascularization, or stent thrombosis recorded, and only one myocardial infarction event by 2 years [53]. Positive vessel remodeling and restoration of vasomotion were also noted, carrying a great deal of promise of $\mathrm{BVS}^{\mathrm{TM}}$ stent. The REVA ${ }^{\mathrm{TM}}$ stent (REVA Medical, San Diego, California, USA) is a stent made of tyrosine poly(desamino tyrosyl-tyrosine ethyl ester) carbonate that metabolizes to amino acids, ethanol, and carbon dioxide. The radiopacity is provided by chemical modifications of tyrosine incorporated iodine with a resorption time of approximately 36 months [54]. Clinical outcomes indicate minimal acute stent recoil and injury, a non-thrombogenic response and complete endothelialization at $30 \mathrm{~d}$. The long term studies also provide encouraging results.

The field of biodegradable metal stents has also gained attention from researchers around the world. Up to now, proposed biodegradable metals can be identified as: (1) Mg-based alloys, (2) pure Fe and Fe-based alloys, (3) metallic zinc, (4) metallic glasses which have been developed from both Mg-based and Fe-based alloys [55]. However, only Mg-based BDS have run to clinical tests. In the physiological environment, $\mathrm{Mg}$ or its alloys corrodes into soluble $\mathrm{Mg}(\mathrm{OH})_{2}, \mathrm{MgCl}_{2}$ and $\mathrm{H}_{2}$ at a fast corrosion rate [56]. The released $\mathrm{Mg}$ ions together with the formation of hydrogen bubbles will lead to the higher local $\mathrm{pH}$ values which may cause chronic inflammatory reactions and blood disorder $[57,58]$. From a mechanical point of view, the Mg-based strut becomes thinner during the process of bio-corrosion, resulting in the loss of strength for scaffolding. Although the impacts of corrosion products on cardiovascular tissues are still not sufficiently investigated and the strength and ductility of Mg-based stents are not always satisfactory, the initial clinical success of the magnesium stent encourages the scientist to focus on the new generation BDS. The $\mathrm{AMS}^{\mathrm{TM}}$ (AMS-1, BIOTRONIK, Berlin, Germany), which is the first metallic biodegradable stent implanted in human is composed of $93 \%$ magnesium and $7 \%$ rare earth metals. The PROGRESS-AMS clinical trial showed that biode- gradable magnesium stents can achieve an immediate angiographic result similar to the result of other metal stents, and can be safely degraded within 4 months [59]. Subsequently, the AMS-INSIGHT clinical trial was carried out and demonstrated that the AMS technology is a safe technique to use for treating peripheral arterial disease though AMS was tested for the treatment of below-the-knee critical limb ischemia [60]. According to the clinical information back to the scientists, the focus of further development of new AMS is on prolonging stent degradation time and introducing drug eluting therapy. In 2010, the clinical evaluation of a biodegradable metallic drug eluting stent for the treatment of vascular disease has begun. This novel device is made of a proprietary magnesium alloy incorporating bioabsorbable polymer loading antiproliferative drug paclitaxel with the aim at reducing neointimal hyperplasia [61]. In May 2011, Biotronik [62] announced positive 6-month results for DREAMS. Among the first 22 patients, there was no cardiac death, no target vessel myocardial infarction and no stent thrombosis, which demonstrate a high safety profile of the novel device. Twelve-month results were reported recently to demonstrate safety and confirm vasomotion, showing the huge potential for DREAMS since it combines deployment and post-dilatation properties and long-term outcomes comparable to DES with the additional benefits of vascular restoration therapy. These promising clinical results show that the feasibility and safety of absorbable magnesium scaffolds and it might be an alternative to polymeric absorbable scaffolds. Fe-based stent does not develop as that rapidly compared with $\mathrm{Mg}$-based stent. The stents made of pure Fe and its alloys are still under evaluations concerning the compatibility of ECs [63] and SMCs [64], toxicity of metabolic or degradable products [65] and gene expression profile [66]. Pre-clinical studies are carried out in the descending aorta of New Zealand rabbits [67] and coronary arteries of porcine [68]. Despite being an interesting alternative candidate for biodegradable implant material, iron mainly reacts like a permanent implant due to the very low degradation rate in physiological media. Since most biodegradable iron stent remained intact after 1 year, intense work has been done on the developing novel Fe-based alloys aiming at both an increase in degradation rate and an improvement in the mechanical properties [69]. The novel Fe-based alloys (Fe-Mn [70], Fe-Mn-Pd [71]) indicate the feasibility of achieving Fe-based BDS with enhanced degradation rate and suitable strength and ductility. Nevertheless, the long-term safety of Fe-based stents and the degradation behavior in vivo are still to be evaluated. What is more, zinc has been examined for the first time as a bioabsorbable cardiovascular stent material in 2013 [72]. Although a systemic evaluation of zinc applied in vascular stent needed to be carried out, the early results indicated that zinc was a promising material that could supplant magnesium as the favored base metal from the aspects of biocorrosion rate and corrosion products in vivo. 


\section{EPC-capture stent}

EPCs are a small population of $\mathrm{CD} 34^{+}$circulating mononuclear cells which was discovered by Asahara et al. [73] in 1997. Since the great in vitro and in vivo differentiation capacity, EPCs have the potential to upregulate the expression of endothelial specific antigens with the ability to migrate to areas of vascular injury and aid in the regeneration of damaged and dysfunctional endothelium [74]. Hence, new perspectives of rapid self-endothelialization were raised up and novel strategie of in vivo endothelialization began to be carried out by immobilizing $\mathrm{CD} 34^{+}$antibody [75]. When the stent is implanted into the body, the EPCs existing in bone narrow and peripheral blood are supposed to be recruited and captured onto the stent surface. Scientists hoped that native endothelium could be reconstructed within a short period of time through this method [76]. At present, many companies and research groups are devoting lots of resources to realize this conceive.

Genous R-stent ${ }^{\mathrm{TM}}$ stent (Orbus Neich, Fort Lauderdale, Florida, USA) is the first commercial vascular stent applying EPC-capture system which received CE mark in 2005. In brief, anti-CD34 antibody is immobilized on the stainless steel substrate in order to attract EPC to the stent, accelerating natural healing to protect against thrombus and minimize restenosis [77]. Up to now, at least ten clinical trials have been carried out in different countries and ongoing trials evaluating the EPC-capture stent have begun throughout the world. In HEALING-FIM and HEALING II clinical trials, no acute or sub-acute stent thrombosis was observed, suggesting the efficacy and safety of EPC-capture technology [78]. Unfortunately, $\mathrm{CD}^{+}{ }^{+}$antibodies are not specific to EPC, there is possibility for an EPC-capture stent to pilot smooth muscle progenitor cells, which in turn result in neointimal proliferation [79]. Therefore, a new application of EPC-capture stent combined with DES technology is under investigation. EPC-capture technique is constructed on Combo Stent ${ }^{\mathrm{TM}}$ (OrbusNeich) together with abluminal low-dose sirolimus and a biodegradable polymer [80]. In May 2012, OrbusNeich [81] announced that 12-month follow-up data from the REMEDEE study showed favorable clinical and safety outcomes involving the use of the company's Combo Dual Therapy Stent when compared to the TAXUS Liberte ${ }^{\circledR}$ paclitaxel-eluting stent. The clinically driven target lesion failure rate was $8.9 \%$ for patients treated with the Combo stent, compared to $10.2 \%$ for those treated with the TAXUS ${ }^{\mathrm{TM}}$. Also the promising outcomes after almost 18 months support the benefits of the dual therapy approach [82].

Currently, capture of EPC and induction of EPCs homing to the stent to realize in-situ rapid endothelialization have been one of the promising strategies applied in the next generation stents. Besides anti $\mathrm{CD}_{3} 4^{+}$antibody, biomolecules such as VE-cadherin [83], DNA [84] or peptide ap- tamers [85], and magnetic nanoparticles [86] have been reported to present the ability to specifically bind to EPC. However, considering the safety, none of these novel systems was tested in clinic. Although the efficacy of these methods have been demonstrated [87], further investigations and more experimental data from in-vitro and in vivo tests are necessary. And we are looking forward to the early trial of these strategies applied in the next generation EPCcapture stent in clinic.

\section{Time sequence functional stent}

Although numerous commercial stents have successfully applied in clinical tests, continuous optimizing properties of stents is still a huge task for the stent designers. With the better understanding of the pathobiology of stenting response, the failure of BMS, DES, BDS and EPC-capture stent is the mismatch between the stent behavior and biological response. For example, the proliferation of SMCs at early stage (several weeks) post-stenting leads to restenosis. Uncompleted endothelialization after drug release at the middle stage (3-6 months) results in the late thrombosis. The fast degradation rate of biodegradable magnesium stent puzzles the scientist. And the rapid endothelialization of EPC-capture stents requires healing within a few days with assist of anti-coagulant drug. Herein, the concept of time sequence functional stent is raised up. Designs of new generation stents not only focus on the property of anti-coagulation, promotion of ECs, inhibition of SMCs and the differentiation of EPC, but also pay more attention on the time sequence at global perspective. This way, the stent possesses the properties that can always match the requirements of biological environment of the host during the time sequence of healing and thus has the ability to overcome the problems above.

At the early stage (several weeks), restenosis is a predominant issue which should be suppressed. Anticoagulation and endothelialization are essential during the middle stage (3-6 months). The late stage ( $>9$ month) should highlight the prevention of toxic ions release and maintain tissue compatibility. From this point, loading anti-coagulant molecules and anti-proliferative drugs needs precise design and synergetic effect. The passive barrier helps the bare stent to be biocompatible with the local vessel tissue. When it comes to BDS, the fast endothelialization and antithrombotic properties are given prior consideration. Suppressing restenosis and late thrombosis at the middle stage requires attention. At the late stage, in the case of fully degradable stents, tissue responses and the metabolism of degradable products need to be evaluated. Otherwise, the controlled behavior of degradation should coordinate with the healing process of stent implantation. The responses and tasks during various periods along the time sequence after stent implantation are shown in Figure 2. These spatio-temporal effects 


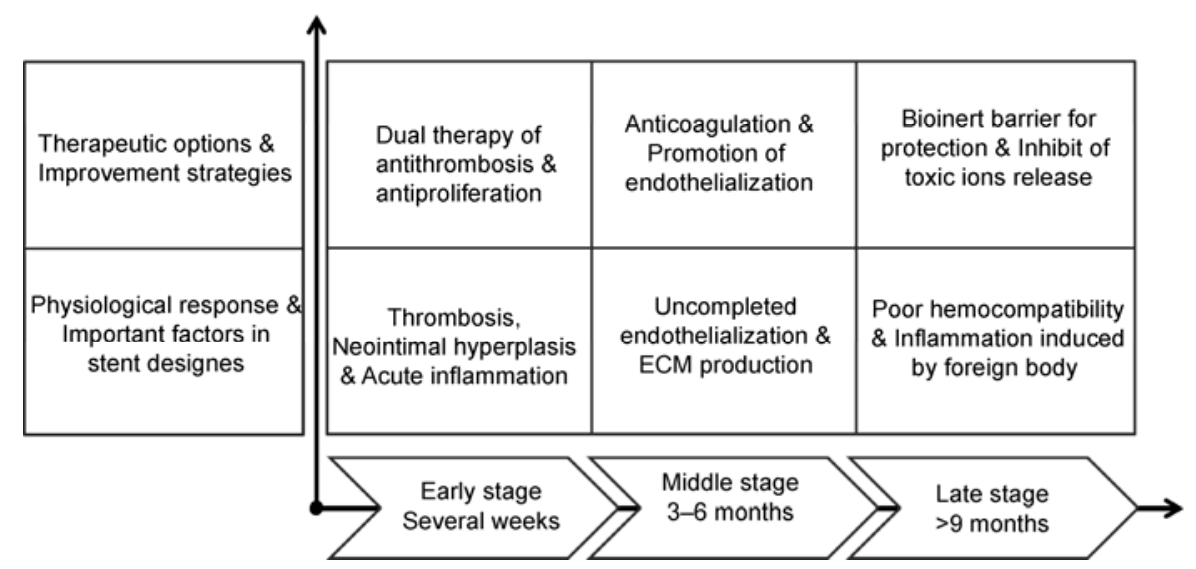

Figure 2 Responses and tasks follow stent implantation.

on the application of cardiovascular stents have been paid more and more attention in recent years. Considering the time-controlled manner, a surface that release therapeutics was achieved by Park group. The controlled release of paclitaxel from the multilayer films successfully led to the apoptosis of SMC in vitro, suggesting the potential application in drug-eluting systems to prevent the restenosis after surgery [88]. Yang et al. [89] construct a kind of stent surface where vascular endothelial growth factor gene and paclitaxel could be eluted at a time sequence scale. This modified stent promote early endothelium healing while inhibit smooth muscle cell proliferation compared with the control TAXUS ${ }^{\mathrm{TM}}$.

However, these designs have not been examined in clinical trials. Also, it is of great difficulties in developing novel ideally stent to solve the problems in all above stages. Authors of this paper have made an attempt on a model stent according to the above described conception and finished its clinical trial. The stent consists of metal stent, titanium oxide film, drug eluting coating (mixture layer of the biodegradable polymer PLGA and rapamycin). At the acute stage the drug release plays the role to suppress restenosis, and then the drug carrier PLGA is degraded and the Ti-O film surface with improved antithrombotic properties is exposed to blood, also supporting the endothelialization. In the long-term stage the Ti-O film coating on the stent prevents the release of deleterious elements from the surface into the surrounding tissue and increased the long-term biocompatibility. The clinical trial was performed as a multicenter randomized study in comparison with a commercial stent. The 150 patients were adopted for each stent group and totally 251 new stents and 240 reference stents were used. One year clinic results showed that the restenosis rate for the new stent was $1.1 \%$ and the late thrombus formation for the new stent was 0 , both significantly lower than the control [90]. It can be concluded that the new stent with the novel structure metal stent/Ti-O film/drug eluting coating has displayed the appropriate properties according to the time stage of the vascular response after stent implantation.
In addition, the realization of time sequence functional stent shows the possibility of constructing novel time-sequence functional stent with improved properties.

\section{Conclusion}

From the bare metal stent to drug eluting stent, and from the biodegradable stent to EPC-capture stent, twenty five years has witnessed the evolution of interventional treatment by vascular stents. Unfortunately, there is still not an ideal design for coronary stents. The relationship between these four general categories of vascular stents is summarized in the triangle from Figure 3. And the directions for improvement of vascular stents could be conjectured. At present, a large number of novel vascular stent models are constructed in the laboratory, emphasizing development of new metallic platforms, new stent bioactive coatings, new drug combinations and new alternative biodegradable substrates. At the same time, a series of clinical trials of different commercial stents are under investigation. The long term efficacy and safety of these stents on the market will be checked by doctors, patients and biomedical engineers.

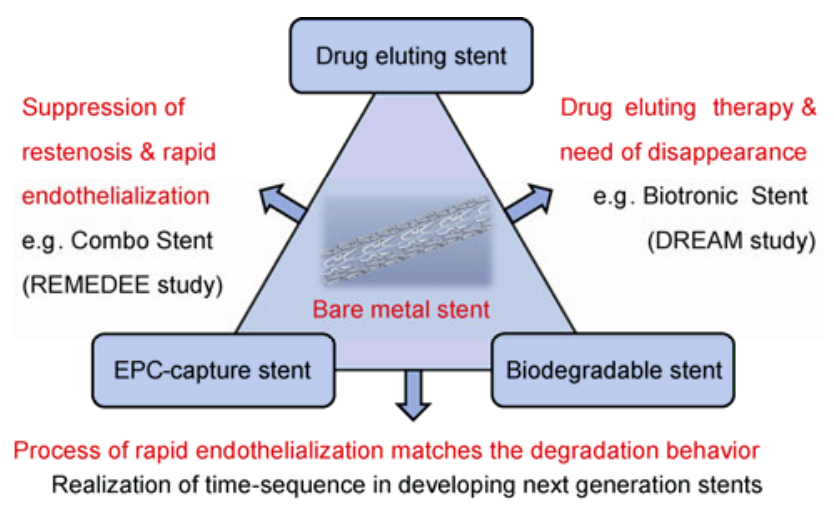

Figure 3 (Color online) Relationship between four general categories of vascular stents. 
This work was supported by the National Basic Research Program of China (2011CB606204) and the National Natural Science Foundation of China (81330031, 81271701).

1 Mackman N. Triggers, targets and treatments for thrombosis. Nature, 2008, 451: 914-918

2 World Health Organization. World Health Statistics, 2012

3 Fontaine B, Fresno C. Vascular Stent. United States Patent, US005314472A, 1994-5-24

4 Alfonso F. Treatment of drug-eluting stent restenosis the new pilgrimage: Quo vadis? J Am Coll Cardiol, 2010, 55: 2717-2720

5 Ong A T, McFadden E P, Regar E, et al. Late angiographic stent thrombosis (LAST) events with drug-eluting stents. J Am Coll Cardiol, 2005, 45: 2088-2092

6 Daemen J, Serruys P W. Drug-eluting stent update 2007: Part I. A survey of current and future generation drug-eluting stents: Meaningful advances or more of the same? Circulation, 2007, 116: 316328

7 Grüntzig A, Schneider H J. The percutaneous dilatation of chronic coronary stenoses-experiments and morphology. Schweiz Med Wochenschr, 1977, 107: 1588

8 Sigwart U, Puel J, Mirkovitch V, et al. Intravascular stents to prevent occlusion and re-stenosis after transluminal angioplasty. N Engl J Med, 1987, 316: 701-706

9 O'Brien B, Carroll W. The evolution of cardiovascular stent materials and surfaces in response to clinical drivers: A review. Acta Biomater, 2009, 5: 945-958

10 Mani G, Feldman M D, Patel D, et al. Coronary stents: A materials perspective. Biomaterials, 2007, 28: 1689-1710

11 Farb A, Weber D K, Kolodgie F D, et al. Morphological predictors of restenosis after coronary stenting in humans. Circulation, 2002, 105: 2974-2980

12 Inoue $\mathrm{T}$, Croce $\mathrm{K}$, Morooka $\mathrm{T}$, et al. Vascular inflammation and repair implications for re-endothelialization, restenosis, and stent thrombosis. JACC Cardiovasc Interv, 2011, 4: 1057-1066

13 Welt F G P, Rogers C. Inflammation and restenosis in the stent era. Arterioscler Thromb Vasc Biol, 2002, 22: 1769-1776

14 Newby A C, Zaltsman A B. Molecular mechanisms in intimal hyperplasia. J Pathol, 2000, 190: 300-309

15 Albiero R, Nishida T, Adamian M. et al. Edge restenosis after implantation of high activity ${ }^{32} \mathrm{P}$ radioactive $\beta$-emitting stents. Circulation, 2000, 101: 2454-2457

16 Leon M B, Teirstein P S, Moses J W, et al. Localized intracoronary gamma-radiation therapy to inhibit the recurrence of restenosis after stenting. N Engl J Med, 2001, 344: 250-256

17 Stone G W, Ellis S G, Cox D A, et al. One-year clinical results with the slow-release, polymer-based, paclitaxel-eluting TAXUS stent the TAXUS-IV trial. Circulation, 2004, 109: 1942-1947

18 Tung R, Kaul S, Diamond G A, et al. Narrative review: Drug-eluting stents for the management of restenosis: A critical appraisal of the evidence. Ann Intern Med, 2006, 144: 913-919

19 Kay I P, Wardeh A J, Kozuma K, et al. Radioactive stents delay but do not prevent in-stent neointimal hyperplasia. Circulation, 2001, 103: $14-17$

20 Martin D M, Boyle F J. Drug-eluting stents for coronary artery disease: A review. Med Eng Phys, 2011, 33: 148-163

21 Wolf K V, Zong Z, Meng J, et al. An investigation of adhesion in drug-eluting stent layers. J Biomed Mater Res A, 2008, 87: 272-281

22 Ranade S V, Miller K M, Richard R E, et al. Physical characterization of controlled release of paclitaxel from the TAXUS ${ }^{\mathrm{TM}} \mathrm{Ex}$ press $2^{\mathrm{TM}}$ drug-eluting stent. J Biomed Mater Res A, 71A: 625-634

23 Windecker S, Remondino A, Eberli F R, et al. Sirolimus-eluting and paclitaxel-eluting stents for coronary revascularization. N Engl J Med, 2005, 353: 653-662

24 Morice M C, Colombo A, Meier B, et al. Sirolimus-vs paclitaxel-eluting stents in de novo coronary artery lesions: The REALITY trial: A randomized controlled trial. JAMA, 2006, 295: 895-904

25 Guagliumi G, Farb A, Musumeci G, et al. Sirolimus eluting stent im- planted in human coronary artery for 16 months: Pathological findings. Circulation, 2003, 107: 1340-1341

26 Virmani R, Guagliumi G, Farb A, et al. Localized hypersensitivity and late coronary thrombosis secondary to a sirolimus-eluting stent should we be cautious? Circulation, 2004, 109: 701-705

27 Pendyala L K, Yin X H, Li J S, et al. The first-generation drug-eluting stents and coronary endothelial dysfunction. JACC-Cardiovasc Interv, 2009, 2: 1169-1177

28 McFadden E P, Stabile E, Regar E, et al. Late thrombosis in drugeluting coronary stents after discontinuation of antiplatelet therapy. Lancet, 2004, 364: 1519-1521

29 Khan W, Farah S, Domb A J. Drug eluting stents: Developments and current status. J Control Release, 2012, 161: 703-712

30 Burke S E, Kuntz R E, Schwartz L B. Zotarolimus (ABT-578) eluting stents. Adv Drug Deliv Rev, 2006, 58: 437-446

31 Sheiban I, Villata G, Bollati M, et al. Next-generation drug-eluting stents in coronary artery disease: Focus on everolimus-eluting stent (Xience V®). Vasc Health Risk Manag, 2008, 4: 31-38

32 Lange R A, Hillis L D, Stone G W, et al. Second-generation drug-eluting coronary stents. N Engl J Med, 2010, 362: 1728-1730

33 Wykrzykowska J J, Onuma Y, Serruys P W. Advances in stent drug delivery: The future is in bioabsorbable stents. Expert Opin Drug Deliv, 2009, 6: 113-126

34 Kukreja N, Onuma Y, Daemen J, et al. The future of drug-eluting stents. Pharmacol Res, 2008, 57: 171-180

35 Waksman R. Drug-eluting stents from bench to bed. Cardiovasc Radiat Med, 2002, 3: 226-241

36 Wessely R. New drug-eluting stent concepts. Nat Rev Cardiol, 2010, 7: 194-203

37 Schwartz R S, Edelman E R, Carter A, et al. Drug-eluting stents in preclinical studies recommended evaluation from a consensus group. Circulation, 2002, 106: 1867-1873

38 Garg S, Swrruys P W. Coronary stents looking forward. J Am Coll Cardiol, 2010, 56(Suppl 10): S43-S78

39 Nakazawa G, Finn A V, Kolodgie F D, et al. A review of current devices and a look at new technology: Drug-eluting stents. Expert Rev Med Devices, 2009, 6: 33-42

40 Grube E, Buellesfeld L. BioMatrix ${ }^{\circledR}$ Biolimus A9 ${ }^{\circledR}$-eluting coronary stent: A next-generation drug-eluting stent for coronary artery disease. Expert Rev Med Devices, 2006, 3: 731-741

41 Abizaid A, Costa J R. New drug-eluting stents an overview on biodegradable and polymer-free next-generation stent systems. Circ Cardiovasc Interv, 2010, 3: 384-393

42 Tsujino I, Ako J, Honda Y, et al. Drug delivery via nano-, micro and macroporous coronary stent surfaces. Expert Opin Drug Deliv, 2007, 4: $287-295$

43 Ohlow M A, von Korn H, Farah A, et al. TCT-601 real-world experience of the polymer-free rapamycin-eluting YUKON-Choice stent: Five-year results from a prospective registry. J Am Coll Cardiol, 2012, 60(Suppl 17): B174

44 Joner M, Finn A V, Farb A, et al. Pathology of drug eluting stents in humans: Delayed healing and late thrombotic risk. J Am Coll Cardiol, 2006, 48: 193-202

45 Ormiston J A, Serruys P W S. Bioabsorbable coronary stents. Circ Cardiovasc Interv, 2009, 2: 255-260

46 Bourantas C V, Onuma Y, Farooq V, et al. Bioresorbable scaffolds: Current knowledge, potentialities and limitations experienced during their first clinical applications. Int J Cardiol, 2012, 167: 11-21

47 Nair L S, Laurencin C T. Biodegradable polymers as biomaterials. Prog Polym Sci, 2007, 32: 762-798

48 Moravej M, Mantovani D. Biodegradable metals for cardiovascular stent application. Int J Mol Sci, 2011, 12: 4250-4270

49 Tamai H, Igaki K, Takafumi T, et al. A biodegradable poly-L-lactic acid coronary stent in the porcine coronary. J Interv Cardiol,1999, 12: $443-450$

50 Tamai H, Igaki K, Kyo E, et al. Initial and 6-month results of biodegradable poly-L-lactic acid coronary stents in humans. Circulation, 2000, 102: 399-404

51 Nishio S, Kosuga K, Igaki K, et al. long-term (>10 years) clinical 
outcomes of first-in-human biodegradable poly-L-lactic acid coronary stents: Igaki-tamai stents. Circulation, 2012, 125: 2343-2353

52 Raoul B, Asgar W A. Biodegradable stents-Where are we in 2009? US cardiol, 2009, 6: 81-84

53 Serruys P W, Ormiston J A. Onuma Y, et al. A bioabsorbable everolimus-eluting coronary stent system (ABSORB): 2-Year outcomes and results from multiple imaging methods. Lancet, 2009, 373: 897-910

54 Onuma Y, Serruys P W. Bioresorbable scaffold: The advent of a new era in percutaneous coronary and peripheral revascularization? Circulation, 2011, 123: 779-797

55 Hermawan H, Dubé D, Mantovani D. Developments in metallic biodegradable stents. Acta Biomater, 2010, 6: 1693-1697

56 Yun Y, Dong Z Y, Lee N, et al. Revolutionizing biodegradable metals. Mater Today, 2009, 12: 22-32

57 Zberg B, Uggowitzer P J, Loeffler J F. MgZnCa glasses without clinically observable hydrogen evolution for biodegradable implants. Nat Mater, 2009, 8: 887-891

$58 \mathrm{Gu} \mathrm{X} \mathrm{N}$, Zheng Y F, Cheng Y, et al. In vitro corrosion and biocompatibility of binary magnesium alloys. Biomaterials, 2009, 30: 484498

59 Erbel R, Di Mario C, Bartunek J, et al. Temporary scaffolding of coronary arteries with bioabsorbable magnesium stents: A prospective, non-randomised multicentre trial. Lancet, 2007, 369: 1869-1875

60 Bosiers M. AMS insight-Absorbable metal stent implantation for treatment of below-the-knee critical limb ischemia: 6-Month analysis. Cardiovasc Interv Radiol, 2009, 32: 424-435

61 Waksman R, Pakala R. Biodegradable and bioabsorbable stents. Curr Pharm Design, 2010, 16: 4041-4051

62 Haude M, Erbel R, Erne P, et al. Safety and performance of the drug-eluting absorbable metal scaffold (DREAMS) in patients with de-novo coronary lesions: 12 Month results of the prospective, multicentre, first-in-man BIOSOLVE-I trial. Lancet, 2013, 381: 836-844

63 Zhu S F, Huang N, Xu L, et al. Biocompatibility of pure iron: In vitro assessment of degradation kinetics and cytotoxicity on endothelial cells. Mat Sci Eng C, 2009, 29: 1589-1592

64 Schaffer J E, Nauman E A, Stanciu L A. Cold-drawn bioabsorbable ferrous and ferrous composite wires: An evaluation of in vitro vascular cytocompatibility. Acta Biomater, 2013, 9: 8574-8584

65 Mueller P P, May T, Perz A, et al. Control of smooth muscle cell proliferation by ferrous iron. Biomaterials, 2006, 27: 2193-2200

66 Purnama A, Hermawan H, Champetier S, et al. Gene expression profile of mouse fibroblasts exposed to a biodegradable iron alloy for stents. Acta Biomater, 2013, 9: 8746-8753

67 Peuster M, Wohlsein P, Brugmann M, et al. A novel approach to temporary stenting: Degradable cardiovascular stents produced from corrodible metal-results 6-18 months after implantation into New Zealand white rabbits. Heart, 2001, 86: 563-569

68 Waksman R O N, Pakala R, Baffour R, et al. Short-term effects of biocorrodible iron stents in porcine coronary arteries. J Interv Cardiol, 2008, 21: 15-20

69 Liu B, Zheng Y F. Effects of alloying elements (Mn, Co, Al, W, Sn, $\mathrm{B}, \mathrm{C}$ and $\mathrm{S}$ ) on biodegradability and in vitro biocompatibility of pure iron. Acta Biomater, 2011, 7: 1407-1420

70 Hermawan H, Purnama A, Dube D, et al. Fe-Mn alloys for metallic biodegradable stents: Degradation and cell viability studies. Acta Biomater, 2010, 6: 1852-1860

71 Schinhammer M, Hänzi A C, Löffler J F, et al. Design strategy for biodegradable Fe-based alloys for medical applications. Acta Biomater, 2010, 6: 1705-1713
72 Bowen P K, Drelich J, Goldman J. Zinc exhibits ideal physiological corrosion behavior for bioabsorbable stents. Adv Mater, 2013, 25: 2577-2582

73 Asahara T, Murohara T, Sullivan A, et al. Isolation of putative progenitor endothelial cells for angiogenesis. Science, 1997, 275: 964967

74 Yoder M C. Defining human endothelial progenitor cells. J Thromb Haemost, 2009, 7(Suppl 1): 49-52

75 Avci-Adali M, Ziemer G, Wendel H P. Induction of EPC homing on biofunctionalized vascular grafts for rapid in vivo self-endothelialization-A review of current strategies. Biotechnol Adv, 2010, 28: 119-129

76 Wendel H P, Avci-Adali M, Ziemer G. Endothelial progenitor cell capture stents-Hype or hope? Int J Cardiol, 2010, 145: 115-117

77 Padfield G J, Newby D E, Mills N L. Understanding the role of endothelial progenitor cells in percutaneous coronary intervention. $\mathbf{J}$ Am Coll Cardiol, 2010, 55: 1553-1565

78 Klomp M, Beijk M A M, de Winter R J. Genous ${ }^{\mathrm{TM}}$ endothelial progenitor cell-capturing stent system: A novel stent technology. Expert Rev Med Devices, 2009, 6: 365-375

79 Yeh E T, Zhang S, Wu H D, et al. Transdifferentiation of human peripheral blood $\mathrm{CD} 34^{+}$-enriched cell population into cardiomyocytes, endothelial cells, and smooth muscle cells in vivo. Circulation, 2003, 108: 2070-2073

80 Granada J F, Inami S, Aboodi M S, et al. Development of a novel prohealing stent designed to deliver sirolimus from a biodegradable abluminal matrix. Circ Cardiovasc Interv, 2010, 3: 257-266

81 OrbusNeich's Combo Dual Therapy Stent ${ }^{\mathrm{TM}}$ Demonstrates Favorable Clinical and Safety Outcomes at 12-Month Follow-Up. OrbusNeich Report, 2012

82 Lee S W L, Lam S C C, Chan K K W, et al. TCT-292 evaluation of neointimal healing and late luminal loss of endothelial progenitor cell capturing sirolimus-eluting (COMBO) stent by optical coherence tomography: The EGO-COMBO dtudy. J Am Coll Cardiol, 2012, 60(Suppl 17): B82

83 Lee J M, Choe W S, Kim B K, et al. Comparison of endothelialization and neointimal formation with stents coated with antibodies against CD34 and vascular endothelial-cadherin. Biomaterials, 2012, 33: 8917-8927

84 Sim D S, Kim Y S, Hong Y J, et al. Original paper: Experience with endothelial progenitor cell capturing aptamers for coating of intracoronary stents in a porcine model. Tissue Eng Regener Med, 2009, 6: $555-561$

85 Veleva A N, Heath D E, Cooper S L, et al. Selective endothelial cell attachment to peptide-modified terpolymers. Biomaterials, 2008, 29: 3656-3661

86 Li Q Y, Tang G H, Xue S H, et al. Silica-coated superparamagnetic iron oxide nanoparticles targeting of EPCs in ischemic brain injury. Biomaterials, 2013, 34: 4982-4992

87 Avci-Adali M, Stoll H, Wilhelm N, et al. In vivo tissue engineering: Mimicry of homing factors for self-endothelialization of bloodcontacting materials. Pathobiology, 2013, 80: 176-181

88 Park S, Bhang S H, La W G, et al. Dual roles of hyaluronic acids in multilayer films capturing nanocarriers for drug-eluting coatings. Biomaterials, 2012, 33: 5468-5477

89 Yang J, Zeng Y, Zhang C, et al. The prevention of restenosis in vivo with a VEGF gene and paclitaxel co-eluting stent. Biomaterials, 2013, 34: 1635-1643

90 Qi P K, Maitz M F, Huang N. Surface modification of cardiovascular materials and implants. Surf Coat Technol, 2013, 233: 80-90

Open Access This article is distributed under the terms of the Creative Commons Attribution License which permits any use, distribution, and reproduction in any medium, provided the original author(s) and source are credited. 